\title{
thelomj
}

Letters Volume-mortality for cystectomy

\section{Centralisation of cancer services vindicated}

BMJ 2010; 340 doi: http://dx.doi.org/10.1136/bmj.c3336 (Published 22 June 2010) Cite this as: BMJ 2010;340:c3336

Paul J Cathcart, academic clinical lecturer in urology1, Jan van der Meulen, professor of clinical epidemiology and director2, Mark Emberton, reader in interventional oncology and clinical director ${ }^{3}$, John Kelly, chair of uro-oncology and chair, NCRI Bladder Clinical Studies Group 4 ${ }^{1}$ University College London Hospital and Clinical Effectiveness Unit, Royal College of Surgeons of England, London WC2A 3PE

${ }^{2}$ Health Services Research Unit, London School of Hygiene and Tropical Medicine and Clinical Effectiveness Unit, Royal College of Surgeons of England, London WC2A 3PE

${ }^{3}$ Royal Free and University College London School of Medicine, University College London and Clinical Effectiveness Unit, Royal College of Surgeons of England, London WC1E 6AU

${ }^{4}$ University College London Hospital and Division of Surgery and Interventional Science, UCL Medical School, University College London, London WC1E 6AU

pjcathcart@hotmail.com

Mayer and colleagues evaluated the relation between volume and mortality for radical cystectomy in England.1 Their finding - that patients undergoing surgery in medium volume hospitals have a poorer outcome than those in low volume hospitals—contrasts with most published data.2 3

A weakness of Mayer and colleagues' study is that they report only short term outcomes after radical cystectomy-namely, inhospital and 30 day mortality. Mortality after cystectomy has been reported to rise significantly beyond 30 days. 4

To evaluate whether hospital volume influences longer term outcomes after cystectomy, we identified patients undergoing radical cystectomy, again within the hospital episode statistics database, but slightly more recently (2002-3 and 2007-8).

Like Mayer and colleagues, we found that 30 day mortality was not statistically different between the hospitals of different volumes (low 3.0\%, medium 2.7\%, and high $2.4 \%$; $\mathrm{P}=0.22$ ). However, when we evaluated 90 day mortality, high volume hospitals significantly outperformed both medium and low volume hospitals (low 7.6\%, medium 6.2\%, high 5.7\%; P=0.007).

The National Institute for Health and Clinical Excellence (NICE) recommends that radical cystectomy be performed only in hospitals doing more than 50 pelvic cancer procedures a year. 5 Although we did not adjust for structural and processes of care as Mayer and colleagues did, our results support this guidance. When outcomes of pelvic cancer surgery are being evaluated, analysis of 30 day mortality may result in erroneous conclusions. 


\section{Notes}

Cite this as: BMJ 2010;340:c3336

\section{Footnotes}

- Competing interests: JK is chair of the NCRI Bladder Clinical Studies Group.

\section{References}

1. Mayer EK, Bottle A, Darzi AW, Athanasiou T, Vale JA. The volume-mortality relation for radical cystectomy in England: retrospective analysis of hospital episode statistics. BMJ2010;340:c1128. (19 March.)

2. .Birkmeyer JD, Siewers AE, Finlayson EV, Stukel TA, Lucas FL, Batista I, et al. Hospital volume and surgical mortality in the United States. N Engl J Med2002;346:1128-37.

3. Joudi FN, Konety BR. The impact of provider volume on outcomes from urological cancer therapy. $J$ Urol2005;174:432-8.

4. Isbarn H, Jeldres C, Zini L, Perrotte P, Baillargeon-Gagne S, Capitanio U, et al. A population based assessment of perioperative mortality after cystectomy for bladder cancer. J Uro/2009;182:70-7. Epub 2009 May 17.

5. National Institute for Clinical Excellence. Improving outcomes in urological cancers. London: NICE, 2002. Available at: www.nice.org.uk/nicemedia/live/10889/28771/28771.pdf. 